\title{
1 Spider lures exploit insect preferences for floral colour and
}

\section{2 symmetry}

4 Thomas E. White ${ }^{1,3}$, Darrell J. Kemp ${ }^{2}$

$6{ }^{1}$ School of Life and Environmental Sciences, The University of Sydney, Sydney, Australia 2106

$7 \quad{ }^{2}$ Department of Biological Sciences, Macquarie University, North Ryde, Australia 2113.

$8 \quad{ }^{3}$ Corresponding author.

10 E-mail: thomas.white@sydney.edu.au

11 Keywords: sensory trap, sensory bias, orb-web spider, prey lure, mimicry

12 Word count (excluding abstract): 3219

13 Number of figures: 3

14 Number of tables: 0

15 Supplementary information: Table S1 


\section{Abstract}

18 Sensory systems can capture only a fraction of available information, which creates opportunities

19 for deceptive signalling. The sensory traps and sensory bias models have proven valuable for

20 explaining how visual systems and environments shape the design of sexual signals, but their

21 application to deceptive signals is largely untapped. Here we use the 'jewelled' orb-web spider

22 Gasteracantha fornicata to experimentally test two longstanding hypotheses for the function of

23 deceptive visual lures. Namely, that they: (1) exploit generalised preferences for conspicuous

24 colouration (sensory bias), or (2) co-opt the otherwise-adaptive foraging response of prey toward

25 flowers (sensory traps). In a field-based study we manipulated the conspicuous dorsal signal of

26 female spiders along two axes — colour pattern and symmetry — to generate a gradient of floral

27 resemblance, and monitored the per-individual consequences for prey interception. As predicted

28 by the traps model, the most attractive phenotypes were those with flower-like radial symmetry

29 and solid colour patterns, and their attractiveness equaled that of wild-type models. These results

30 demonstrate that deceptive orb-web spider lures function, in part, as inter-kingdom sensory traps

31 via floral mimicry, and support the broader extension of sensory-based models to deceptive

32 signalling contexts. 


\section{Introduction}

Visual communication is ubiquitous, and the demands of effective information-exchange

41 have driven diverse outcomes (Maia et al. 2013; Thoen et al. 2014; Dalrymple et al. 2015).

42 Understanding this diversity requires examining the relationship between signals, environments,

43 and sensory systems, for which the sensory traps and bias models - under the umbrella of sensory

44 drive — have proven valuable (among a suite of related models; Christy 1995; Endler 1992; Endler

45 and Basolo 1998; West-Eberhard 1979). According to the sensory trap model, signals evolve under

46 a model-mimic dynamic to co-opt receiver responses that function adaptively in otherwise

47 unrelated behavioural contexts (Christy et al. 2003). This model accounts for how the design of

48 sexual signals, for example, may be shaped by how potential mates detect or recognize food items

49 (Rodd et al. 2002) or shelter (Christy et al. 2003). The sensory bias model, by contrast, emphasizes

50 how underlying sensory and/or perceptual biases may present opportunities for exploitation and

51 hence drive signal evolution (Basolo and Endler 1995; Ryan and Cummings 2013). The elaborate

52 fins of male swordtails present a canonical example (Basolo 1990), having evolved in response to

53 a pre-existing female bias toward such structures (Basolo 1990; Basolo 1995). Each of these two

54 models has robust empirical support in the context of sexual signalling, however much remains to

55 be learned about their ability to explain signal evolution more broadly.

Visual luring is a widespread predatory strategy and is particularly common among sit-

57 and-wait predators. Orb-web spiders are a model group, with many species combining striking

58 body colours and patterns to actively attract insect prey to the web (Tso et al. 2004; Chuang et al.

59 2007a; White and Kemp 2015). The question of why such conspicuous deceptive signals are

60 attractive to insect viewers has been the focus of considerable attention (Tso et al. 2004; Chuang

61 et al. 2007b; Rao et al. 2015; Goncalves and Gawryszewski 2017; White and Kemp 2017). Two 
62 hypotheses predominate, which informally mirror the bias and traps models; namely, that lures (1)

63 exploit innate colour preferences, or (2) co-opt the foraging response of prey toward flowers.

64 Empirical support for these hypotheses is presently limited to observational and correlative data,

65 and hence remains equivocal (e.g., Tso et al. 2004; Chuang et al. 2007b; Goncalves and

66 Gawryszewski 2018; White et al. 2017). Formalising these hypotheses within the models of

67 sensory theory offers a promising path to progress and may prove reciprocally beneficial in guiding

68 future studies of deceptive signalling.

69 Whereas predictions from the bias and traps models overlap to some degree, their core

70 predictions as applied to deceptive lures can be neatly partitioned (White \& Kemp 2015). If

71 conspicuous visual lures are exploiting receivers' sensory biases, then the most likely perceptual

72 target is colour. The insect prey of luring predators are taxonomically diverse, albeit with an

73 overrepresentation of pollinating flies and bees (Nentwig 1985; Nentwig 1987; O’Hanlon et al.

74 2014a). Strong innate preferences for (human-perceived) yellows and whites are well documented

75 (Kay 1976; Lunau 1988; Lunau and Maier 1995), which parallels a notably biased distribution of

76 these colours among predator lures (White and Kemp 2015). A standing prediction under the bias

77 model, then, is that the expression of preferred colours among deceptive signallers should predict

78 their attractiveness to potential prey. The traps hypothesis, by contrast, suggests that lures are

79 exploiting an otherwise-adaptive attraction to flowers in a dynamic more closely akin to floral

80 mimicry. When foraging, pollinating insects integrate the aforementioned colour preferences with

81 information on different forms of floral symmetry, which they can readily perceive and express

82 preferences for (as contrasted with asymmetry, which is aversive: Chittka and Raine 2006; Kay

83 1976; Lehrer et al. 1995; Lunau and Maier 1995; Giurfa et al. 1996), and radial symmetry is both

84 the most ancient and common form showcased among angiosperms (Crane et al. 1995; Neal et al. 
85 1998; Endress 2001). Accumulating evidence for the predicted resemblance between lures and

86 these features of sympatric flowers supports this mimetic view (Tso et al. 2004; Goncalves and

87 Gawryszewski 2017; White et al. 2017). The key untested prediction, however, is that lures should

88 co-opt the prey's natural response toward flowers. The strongest evidence to date comes from the

89 orchid mantis, which resembles sympatric flowers and presents a more attractive signal to

90 pollinators (O'Hanlon et al. 2013; O'Hanlon et al. 2014a; O'Hanlon et al. 2014b). Although this

91 presents a compelling example of pollinator deception, the restricted range of experimental stimuli

92 offered to viewers in the key assay presents a challenge to unambiguously distinguishing between

93 the traps and (more permissive) bias explanations.

94 Here we sought to formalise and test these adaptive hypotheses for deceptive signalling

95 using the jewelled orb-web spider Gasteracantha fornicata (supplementary Fig. S1). Females of

96 the species are colour polymorphic sit-and-wait predators, whose striking yellow- or white-and-

97 black banded abdomens lure prey — primarily pollinating Diptera and Hymenoptera — to their

98 webs (Hauber 2002; Kemp et al. 2013; White and Kemp 2016). To distinguish between the traps

99 and bias hypotheses we manipulated the appearance of wild female G. fornicata in their natural

100 habitats along two independent axes - colour and symmetry (Fig. 1). Our manipulations consisted

101 of nine different treatments (including the wild-type) that encompassed the full-factorial

102 combination of three levels of colour and three levels of symmetry. The sum of treatments

103 represented an approximate gradient of floral resemblance, thereby affording clear predictions for

104 relative attractiveness under a (generalized) sensory trap hypothesis (i.e., the x-axis of Fig. 2).

105 Predicted attractiveness under the sensory bias model is however different because the main vector

106 of attractiveness in this case should relate to stimulus color alone (the y-axis of Fig. 2). We 
107 evaluated these predictions according to realized prey capture rates of wild, free-ranging spiders

108 randomly assigned among the nine treatment stimuli.

\section{Methods}

114 symmetric shapes, in a fully factorial combination of solid black, black-and-yellow banded, and

115 solid yellow patterns $(n=17-29$ each; Fig. 1). We manipulated the appearance of spiders by fixing

116 a painted cardboard model (Quill 180 gsm paper) corresponding to a given treatment (Fig. 1) to

117 each individual's otherwise flat dorsal abdomen using a ca. $5 \mathrm{~mm}^{2}$ square of double-sided tape.

118 Importantly, we controlled the proportionate size of stimuli in each symmetry-class to ensure an

119 equal area of colour coverage. That is, all solid-yellow models displayed approximately the same

120 total amount of yellow (ca. $81 \mathrm{~mm}^{2}$ ), all striped models had equal amounts of yellow and black

121 (ca. $40 \mathrm{~mm}^{2}$ ), and all black models displayed the same amount of black (ca. $81 \mathrm{~mm}^{2}$ ). We used

122 Derivan Matisse Yellow-Mid AZO Series 2 paint to imitate the yellow colouration of G. fornicata,

123 which has previously been spectrally matched for this purpose using standard methods and is also

124 a known match to sympatric insect-pollinated flora (Maia et al. 2019; White and Kemp 2017). In

125 addition to the nine primary treatments we included a further control in which spiders were

126 unmanipulated save for a square of double-sided tape on their ventrum. Although G. fornicata are

127 colour polymorphic, we used only yellow colouration in all treatments for simplicity and 
128 manipulated both 'white' and 'yellow' individuals in the field. There is some evidence for

129 microhabitat differentiation between G. fornicata morphs (White and Kemp 2016), but our

130 application of treatments was randomised and hence simply contributes unbiased residual variation

131 (i.e., noise). The extent of any microhabitat effects therefore adds conservatism to our focal

132 contrasts.

133 To estimate prey interceptions as a key component of fitness we used a transect-based

134 method comparable to one previously used in this system (White 2017). After applying the

135 cardboard models, we recorded the presence of new prey and/or web damage at 30 minute intervals

136 for 4 hours, either in a morning (0800-1200) or, less often, afternoon (1300-1700) session. Abiotic

137 confounds (such as web damage by wind-blown debris) may inflate true interception rates, but

138 such effects would again be randomly distributed across treatments and simply inflate residual

139 variation. Spiders whose webs that sustained $>50 \%$ damage during an observation period were

140 taken to indicate gross environmental disturbance and were excluded $(\mathrm{n}=12)$ as well as those

141 whose model did not remain affixed $(n=4)$. All work took place in November 2018 across

142 populations spanning Cairns to Port Douglas, Queensland, Australia. The observer (TEW) could

143 not possibly be blind in regard to treatments, but the unambiguous response variable should work

144 to ameliorate unconscious bias.

\section{Statistical analyses}

147 To validate the baseline efficacy of the phenotypic manipulations, we first tested for

148 differences in prey interceptions between the wild-type models of G. fornicata (biradial striped;

149 Fig. 1 centre) and unmanipulated spiders using a generalised linear mixed-effects model (GLMM). 
150 We specified interception rate (mean interceptions / 30 minutes) as the Gaussian response

151 following confirmation of the normality within groups, and treatment (presence/absence) as a main

152 effect, with diel session (morning/afternoon) as a random covariate to account for any systematic

153 differences associated with diel insect activity.

154 For the central tests we used a GLMM with interception rate (mean interceptions / 30

155 minutes) as the response, as above. We specified an interaction between colour

156 (black/striped/solid) and symmetry (asymmetric/biradial/radial) and their main effects, and

157 included diel session (morning/afternoon) as a random covariate. We then used Tukey post-hoc

158 contrasts to test for pairwise differences across all treatment combinations. Should the sensory bias

159 model best explain the attractiveness of phenotypes we predict a main effect of colour alone (Fig.

160 2a). In contrast, the sensory traps hypothesis predicts an interaction between colour and symmetry,

161 with post-hoc tests revealing grouped differences in the manner specified in Figure $2 b$ (and as

162 discussed above). Summary statistics reported below are pooled means \pm standard deviations of

163 prey interceptions rates (interceptions / 30 minutes). All analyses were run in R v. 3.5.2 (R Core

164 Team 2018) using 'nlme' (Pinheiro et al. 2018) for linear mixed modelling and 'multcomp'

165 (Hothorn et al. 2008) for multiple comparisons.

167 Data availability

168 All data and code will be made persistently available via Github and Zenodo upon acceptance. 


\section{Results}

171 We found no difference in prey interception rates between control Gasteracana. fornicata

172 and wild-type models $\left(\mathrm{F}_{1,41}=0.65, p=0.43, \mathrm{R}^{2}=0.02\right)$. The vanishingly small effect size between

173 each group moreover supports the absence of any biologically-relevant consequence of handling.

174 For the main test, we found an interactive effect of colour and symmetry on prey interception rates

$175 \quad\left(\mathrm{~F}_{4,218}=4.12, p=<0.01\right.$, conditional $\left.\mathrm{R}^{2}=0.54\right)$, as well as main effects of colour $\left(\mathrm{F}_{2,218}=107.40\right.$,

$176 p=<0.01)$ and symmetry $\left(\mathrm{F}_{2,218}=15.08, p=<0.01\right)$. Pairwise contrasts (supplementary table $\left.\mathrm{S} 1\right)$

177 revealed considerable variation in prey interception rates between treatments, with three distinct

178 phenotypic groupings (Fig 3). Spiders assigned to black control treatments intercepted prey less

179 frequently than all others $(0.84 \pm 0.77)$, while both striped- and solid-coloured asymmetric

180 phenotypes had greater capture success $(1.92 \pm 0.70)$. The highest rates of prey interception were

181 shared by radially and biradially symmetric treatments across both striped- and solid-coloured

182 phenotypes $(2.86 \pm 0.89)$

183

\section{Discussion}

Visual lures are a striking adaptation for predation, but the mechanism of deception is

186 poorly resolved. Here we manipulated the phenotypes of the jewelled spider Gasteracantha

187 fornicata along an approximate gradient of floral resemblance to test whether deceptive lures are

188 exploiting simple colour-biases, or co-opting foraging preferences, in prey. As predicted by the

189 sensory traps model (Fig. 2, x axis), we found equivalently heightened prey interception rates

190 between the natural phenotype and the biradially symmetric, solid-yellow (most 'floral') model 
191 (Fig. 3). In contrast, the sheer coverage of yellow colouration on models was not solely predictive

192 of prey interceptions as expected under a bias explanation (that is, the effect of colour manifested

193 via an interaction with symmetry). Control tests suggest that the manipulations were effective and

194 highly specific in consequence, with no difference in interception rates between the wild-type

195 model and unmanipulated spiders, and significant differences between black models and all others

196 (Fig. 3; supplementary table S1). In sum, our results suggest that female G. fornicata co-opt the

197 foraging responses of prey toward flowers, in a deceptive inter-kingdom sensory trap.

199 Gasteracantha fornicata are unlikely to be a simple mimic of any one sympatric flower. Rather,

200 the signals of spiders are likely presenting a combination of visual cues that are shared by local

201 flowers including, but not limited to, the spectral, spatial, and symmetric properties of patterns

202 (O'Hanlon et al. 2014a; O'Hanlon et al. 2014b; White et al. 2017). This accords with known

203 features of visual processing among well-studied insects in which local cues such as (in order of

204 prioritisation) colour, modulation, shape, area, and orientation are weighed and integrated to guide

205 the choice and classification of stimuli (Giurfa et al. 1995; Horridge and Zhang 1995; Giurfa et al.

206 1996; Horridge 2007). These cues can be readily generalised to novel contexts (Stach et al. 2004),

207 and their relative importance may vary during assessments of mimetic accuracy (e.g. colour

208 similarity may prioritised over shape; Kazemi et al. 2014) or with cognitive load (e.g. in speed-

209 accuracy tradeoffs; Chittka \& Osorio 2007). This offers a basis for deceptive signal efficacy among

210 luring predators despite their human-subjective distinctiveness from flowers. That is, lures may

211 need only present an ensemble of a few salient cues, rather than a faithful analogue of floral signals,

212 to exploit the foraging response of insect prey (discussed further below). This possibility is further

213 enabled by the phenotypic diversity of sympatric flora, which present a suite of shapes, 
214 symmetries, and colour patterns from which deceptive signallers may draw (see White et al. 2017

215 for data relevant to G. fornicata specifically). Our finding that colour alone was attractive to

216 insects, yet moreso when combined with floral symmetry cues, is consistent with such a view (Fig.

2173 ), though awaits closely controlled behavioural work to test in detail.

218 While the presence of colour in any form was associated with improved attractiveness, the 219 colour pattern — be it solid or striped — had no further effect (Fig. 3). There are two plausible

220 explanations for the lack of a pattern effect. One is that the stripes cannot be resolved at meaningful

221 distances, and a striped pattern would instead only generate a subtly duller, though still 'solid',

222 signal that is functionally equivalent to their block-coloured counterparts. Although the stripes are

223 indeed likely to be resolved only at close distances by typical fly and bee viewers (Land 1997),

224 past work has shown that interception rates are directly modified by the orientation of the stripes

225 of G. fornicata in the web (White 2017), thereby establishing the discriminability of the patterns

226 at relevant viewing distances. A simple alternative, related to the above, is that both striped and

227 solid variants present attractive cues to viewers that are shared by flowers. Solid colours are typical

228 among flowers, though some $33 \%$ of radially symmetric and $14 \%$ of bilaterally symmetric species

229 also present patterned 'floral guides' (Dafni and Giurfa 1999). Such guides take the form of

230 repeated stripes and/or radiating elements, which serve to draw pollinators to the location of nectar

231 and pollen centers (Dafni and Kevan 1996; Dafni and Giurfa 1999). The banded pattern of G.

232 fornicata and our striped, radial model are thus unlikely to be entirely novel to experienced

233 receivers and may merely present another cue that pollinators recognise as broadly 'floral'.

234 The role of colour in visual deception is widespread, and our results support the extension

235 of sensory models to formalise the study of its causes and predicted consequences more generally.

236 The dynamic displays of crab spiders (Heiling et al. 2003), red rims of pitcher plants (Schaefer 
and Ruxton 2008), and decorated webs of spiders (Herberstein et al. 2000) are striking examples, though identifying the underlying mechanism in each case has proven difficult (Herberstein et al. 2000; Schaefer and Ruxton 2009). Our results reiterate the well understood necessity of considering the perspective of receivers, since human-subjective assessments of similarity are a

241 poor guide to the existence and extent of mimicry (Fig. 3). Though our wild-type and 'floral' spider

242 models bear little human-subjective resemblance, our results are consistent with the view that they

243 converge at some stage of sensory processing in insect viewers to elicit a shared foraging response

244 (as noted above). This accords with evidence from sexual signalling systems in which the co245 option of food detection pathways underlies the attractiveness and early evolution of male sexual

246 ornaments, such as the yellow caudal bands of male swordtail characins (Garcia \& Ramirez 2005;

247 Rodd et al. 2002). Interestingly, once such signals become common within a population, receivers

248 may 'escape' the sensory trap via selection for increased response thresholds or improved

249 discriminability (Garcia \& Ramirez 2005). We may predict a similar course in luring systems,

250 though the consequences for signal evolution will diverge due to differences in the alignment of

251 interests between signallers and receivers. In sexual contexts the interests of both parties are

252 broadly aligned toward reproduction. Although selection may favour the partitioning of receivers'

253 feeding and sexual responses through improved discrimination of mimetic traps, they will

254 ultimately respond positively to both sexual and foraging cues (Basolo and Endler 1995; Ryan and

255 Cummings 2013). With respect to signallers, a known consequence is a shift toward signal honesty

256 which also reduces the foraging costs to receivers of responding to deceptive cues (Garcia \&

257 Ramirez 2005). Luring systems, in contrast, cannot follow such a trajectory since they are entirely

258 antagonistic. Thus while selection for improved discrimination and response thresholds in

259 receivers is a predictable outcome, the consequences for deceptive, as opposed to sexual, signal 
260 evolution will diverge. Possible outcomes include selection for improved mimetic fidelity via the

261 integration of new cues and/or refinement of existing ones (e.g. a move toward closer spectral or

262 morphological resemblance to models), a shift toward dietary specialisation or generalisation

263 depending on the composition of available prey (and their foraging preferences), and/or the

264 evolution of signal polymorphism if available prey and models are diverse enough to generate

265 multiple fitness optima (Kazemi et al. 2014; Kikuchi \& Pfenning 2013; White \& Kemp 2016).

266 These are intriguing avenues for future work and highlight the reciprocal promise of luring systems

267 for fueling both empirical insight and theoretical development.

269 Acknowledgments

270 TEW thanks Elizabeth Mulvenna and Cormac White for their endless support.

\section{Funding}

273 None to report. 


\section{References}

275 Basolo, A. L. 1990. Female preference predates the evolution of the sword in swordtail fish.

276 Science 250:808-810.

277 Basolo, A. L. 1995. Phylogenetic evidence for the role of a pre-existing bias in sexual selection.

278 Proceedings of the Royal Society of London. Series B: Biological Sciences 259:307-311.

279 Basolo, A. L., and J. A. Endler. 1995. Sensory biases and the evolution of sensory systems. Trends 280 in Ecology \& Evolution 10:489-489.

281 Chittka, L., and N. E. Raine. 2006. Recognition of flowers by pollinators. Current opinion in plant 282 biology 9:428-435.

283 Chittka, L., and Osorio, D. 2007. Cognitive dimensions of predator responses to imperfect 284 mimicry. PLoS Biology, 5(12), e339.

285 Christy, J., P. Backwell, and U. Schober. 2003. Interspecific attractiveness of structures built by 286 courting male fiddler crabs: Experimental evidence of a sensory trap. Behavioral Ecology and 287 Sociobiology 53:84-91.

288 Christy, J. H. 1995. Mimicry, mate choice, and the sensory trap hypothesis. The American 289 Naturalist 146:171-181.

290 Chuang, C. Y., E. C. Yang, and I. M. Tso. 2007a. Deceptive color signaling in the night: A 291 nocturnal predator attracts prey with visual lures. Behavioral Ecology 19:237-244.

292 Chuang, C.-Y., E.-C. Yang, and I.-M. Tso. 2007b. Diurnal and nocturnal prey luring of a colorful 293 predator. Journal of Experimental Biology 210:3830-3837.

294 Crane, P. R., E. M. Friis, and K. R. Pedersen. 1995. The origin and early diversification of 295 angiosperms. Nature 374:27.

296 Dafni, A., and M. Giurfa. 1999. The functional ecology of floral guides in relation to insect's 297 behaviour and vision. In Evolutionary theory and processes: Modern perspectives (pp. 363-383). 298 Springer. 
299 Dafni, A., and P. G. Kevan. 1996. Floral symmetry and nectar guides: Ontogenetic constraints 300 from floral development, colour pattern rules and functional significance. Botanical Journal of the 301 Linnean Society 120:371-377.

302 Dalrymple, R., D. J. Kemp, H. Flores-Moreno, S. Laffan, T. White, F. Hemmings, M. Tindall, et 303 al. 2015. Birds, butterflies and flowers in the tropics are not more colourful than those in higher 304 latitudes. Global Ecology and Biogeography 848-860.

305 Endler, J. A. 1992. Signals, signal conditions, and the direction of evolution. American Naturalist 306 S125-S153.

307 Endler, J. A., and A. L. Basolo. 1998. Sensory ecology, receiver biases and sexual selection. 308 Trends in Ecology \& Evolution 13:415-420.

309 Endress, P. K. 2001. Evolution of floral symmetry. Current opinion in plant biology 4:86-91.

310 Garcia, C. M., Ramirez, E. 2005. Evidence that sensory traps can evolve into honest signals. Nature $311434: 501-5$

312 Giurfa, M., W. Backhaus, and R. Menzel. 1995. Color and angular orientation in the discrimination 313 of bilateral symmetric patterns in the honeybee. Naturwissenschaften 82:198-201.

314 Giurfa, M., B. Eichmann, and R. Menzel. 1996. Symmetry perception in an insect. Nature 382:458.

315 Goncalves, N. X., and F. M. Gawryszewski. 2018. Prey and predators perceive orb-web spider 316 conspicuousness differently: evaluating alternative hypotheses for color polymorphism evolution. 317 Current Zoology.

318 Hauber, M. E. 2002. Conspicuous colouration attracts prey to a stationary predator. Ecological 319 Entomology 27:686-691.

320 Heiling, A. M., M. E. Herberstein, and L. Chittka. 2003. Pollinator attraction: Crab-spiders 321 manipulate flower signals. Nature 421:334-334.

322 Herberstein, M., C. Craig, J. Coddington, and M. Elgar. 2000. The functional significance of silk 323 decorations of orb-web spiders: A critical review of the empirical evidence. Biological Reviews 324 of the Cambridge Philosophical Society 75:649-669. 
325 Horridge, A. 2007. The preferences of the honeybee (Apis mellifera) for different visual cues

326 during the learning process. Journal of insect physiology 53:877-889.

327 Horridge, G. A., and S. W. Zhang. 1995. Pattern vision in honeybees (Apis mellifera): Flower-like 328 patterns with no predominant orientation. Journal of Insect Physiology 41:681-688.

329 Hothorn, T., F. Bretz, and P. Westfall. 2008. Simultaneous inference in general parametric models. 330 Biometrical Journal (Vol. 50, pp. 346-363).

331 Kay, Q. 1976. Preferential pollination of yellow-flowered morphs of Raphanus raphanistrum by 332 Pieris and Eristalis spp. Nature 230-232.

333 Kazemi, B., Gamberale-Stille, G., Tullberg, B. S., and Leimar, O. 2014. Stimulus salience as an 334 explanation for imperfect mimicry. Current Biology, 24(9), 965-969.

335 Kemp, D. J., C. Holmes, B. C. Congdon, and W. Edwards. 2013. Color polymorphism in spiny 336 spiders (Gasteracantha fornicata): Testing the adaptive significance of a geographically clinal 337 lure. Ethology 1126-1137.

338 Kikuchi, D. W., and Pfennig, D. W. 2013. Imperfect mimicry and the limits of natural 339 selection. The Quarterly review of biology, 88(4), 297-315.

340 Land, M. F. 1997. Visual acuity in insects. Annual review of entomology 42:147-177.

341 Lehrer, M., G. A. Horridge, S. Zhang, and R. Gadagkar. 1995. Shape vision in bees: Innate 342 preference for flower-like patterns. Phil. Trans. R. Soc. Lond. B 347:123-137.

343 Lunau, K. 1988. Innate and learned behaviour of flower-visiting hoverflies-flower-dummy 344 experiments with Eristalis pertinax (scopoli) (Diptera, syrphidae). Zool. Jb. Physiol. 92:487-499.

345 Lunau, K., and E. J. Maier. 1995. Innate colour preferences of flower visitors. Journal of 346 Comparative Physiology A 177:1-19.

347 Maia, R., D. R. Rubenstein, and M. D. Shawkey. 2013. Key ornamental innovations facilitate 348 diversification in an avian radiation. Proceedings of the National Academy of Sciences 110, 349 10687-10692. 
350 Maia, R., Gruson, H., Endler, J. A., \& White, T. E. (2019). pavo 2: new tools for the spectral and 351 spatial analysis of colour in R. Methods in Ecology and Evolution 10, 1097-1107.

352 Neal, P. R., A. Dafni, and M. Giurfa. 1998. Floral symmetry and its role in plant-pollinator 353 systems: Terminology, distribution, and hypotheses. Annual Review of Ecology and Systematics $354 \quad 29: 345-373$.

355 Nentwig, W. 1985. Prey analysis of four species of tropical orb-weaving spiders (araneae: 356 Araneidae) and a comparison with araneids of the temperate zone. Oecologia 66:580-594.

357 Nentwig, W. 1987. The prey of spiders. In Ecophysiology of spiders (pp. 249-263). Springer, 358 USA.

359 O'Hanlon, J. C., G. I. Holwell, and M. E. Herberstein. 2014a. Pollinator deception in the orchid 360 mantis. The American naturalist 183:126-132.

361 O’Hanlon, J. C., G. I. Holwell, and M. E. Herberstein. 2014b. Predatory pollinator deception: Does 362 the orchid mantis resemble a model species? Current Zoology 60.

363 O'Hanlon, J., D. Li, and Y. Norma-Rashid. 2013. Coloration and morphology of the orchid mantis 364 Hymenopus coronatus (mantodea: Hymenopodidae). Journal of Orthoptera Research 22:35-44.

365 Pinheiro, J., D. Bates, S. DebRoy, D. Sarkar, and R Core Team. 2018. nlme: Linear and nonlinear 366 mixed effects models.

367 Rao, D., E. Castaneda-Barbosa, N. Nunez-Beverido, and F. Diaz-Fleischer. 2015. Foraging 368 benefits in a colour polymorphic neotropical orb web spider. Ethology 121:187-195.

369 R Core Team. 2018. R: A language and environment for statistical computing. R Foundation for 370 Statistical Computing, Vienna, Austria.

371 Rodd F. H., Hughes K. A., Grether G.F., and Baril C. T. (2002) A possible non-sexual origin of 372 mate preference: are male guppies mimicking fruit? Proceedings of the Royal Society of 373 London (B) 269 (1490):475-481.

374 Ryan, M. J., and M. E. Cummings. 2013. Perceptual biases and mate choice. Annual Review of 375 Ecology, Evolution, and Systematics 44:437-459. 
376 Schaefer, H. M., and G. D. Ruxton. 2008. Fatal attraction: Carnivorous plants roll out the red carpet

377 to lure insects. Biology Letters 4:153-155.

378 Schaefer, H. M., and G. D. Ruxton. 2009. Deception in plants: Mimicry or perceptual exploitation?

379 Trends in Ecology \& Evolution 24:676-685.

380 Stach, S., J. Benard, and M. Giurfa. 2004. Local-feature assembling in visual pattern recognition 381 and generalization in honeybees. Nature 429:758.

382 Thoen, H. H., M. J. How, T.-H. Chiou, and J. Marshall. 2014. A different form of color vision in 383 mantis shrimp. Science 343:411-413.

384 Tso, I.-M., C.-W. Lin, and E.-C. Yang. 2004. Colourful orb-weaving spiders, Nephila pilipes, 385 through a bee's eyes. Journal of Experimental Biology 207:2631-2637.

386 West-Eberhard, M. J. 1979. Sexual selection, social competition, and evolution. Proceedings of 387 the American Philosophical Society 123:222-34.

388 White, T. E. 2017. Jewelled spiders manipulate colour-lure geometry to deceive prey. Biology 389 letters 13:20170027.

390 White, T. E., R. L. Dalrymple, M. E. Herberstein, and D. J. Kemp. 2017. The perceptual similarity 391 of orb-spider prey lures and flower colours. Evolutionary Ecology 1:1-20.

392 White, T. E., and D. J. Kemp. 2015. Technicolour deceit: A sensory basis for the study of colour393 based lures. Animal Behaviour 105:231-243.

394 White, T. E., and D. J. Kemp. 2016. Colour polymorphic lures target different visual channels in 395 prey. Evolution 70, 1398-1408.

396 White, T. E., and D. J. Kemp. 2017. Colour polymorphic lures exploit innate preferences for 397 spectral versus luminance cues in dipteran prey. BMC evolutionary biology 17:191 

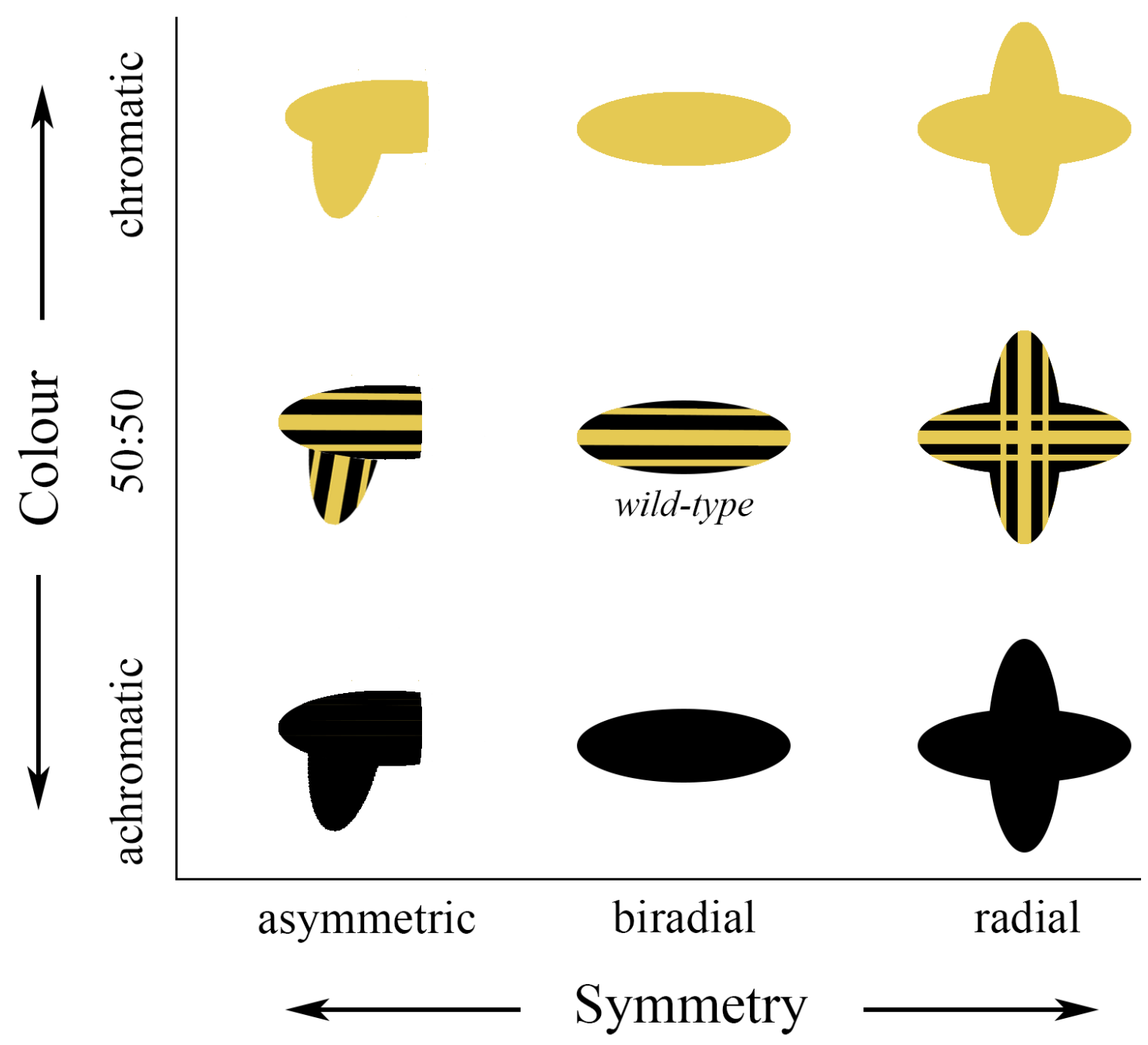

415 Figure 1: The colour pattern manipulations as applied to naturally-occurring female specimens of

416 G. fornicata. The aim was to represent an approximate gradient of floral resemblance from most 417 flower-like (top right) to least (bottom left), while including a wild-type model (center; also see 418 supplementary fig. S1). 


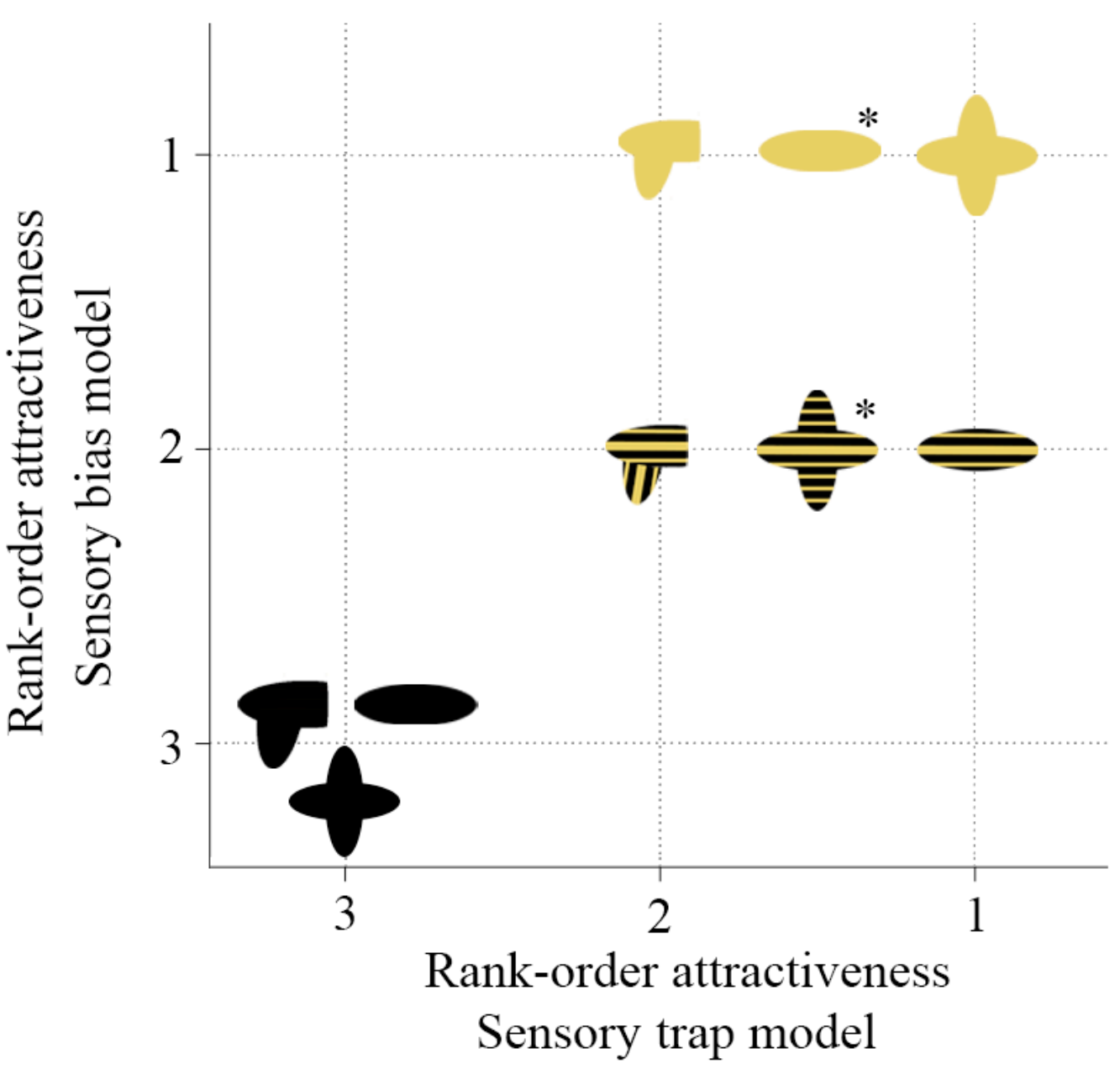

434 Figure 2: The predicted attractiveness of artificial phenotypes under the traps and bias models of 435 signal evolution. If lures are exploiting general colour-biases, the attractiveness of models should 436 simply be predicted by yellow coverage. If, in contrast, lures are floral sensory traps, then the 437 natural phenotype should be as equally attractive as the most 'flower-like' phenotype. Note that 438 solid biradial and striped radial models (asterisked) are of intermediate rank on the $\mathrm{x}$ axis because 439 it is difficult to derive unambiguous predictions for their rank-order attractiveness under the 440 sensory traps model. 


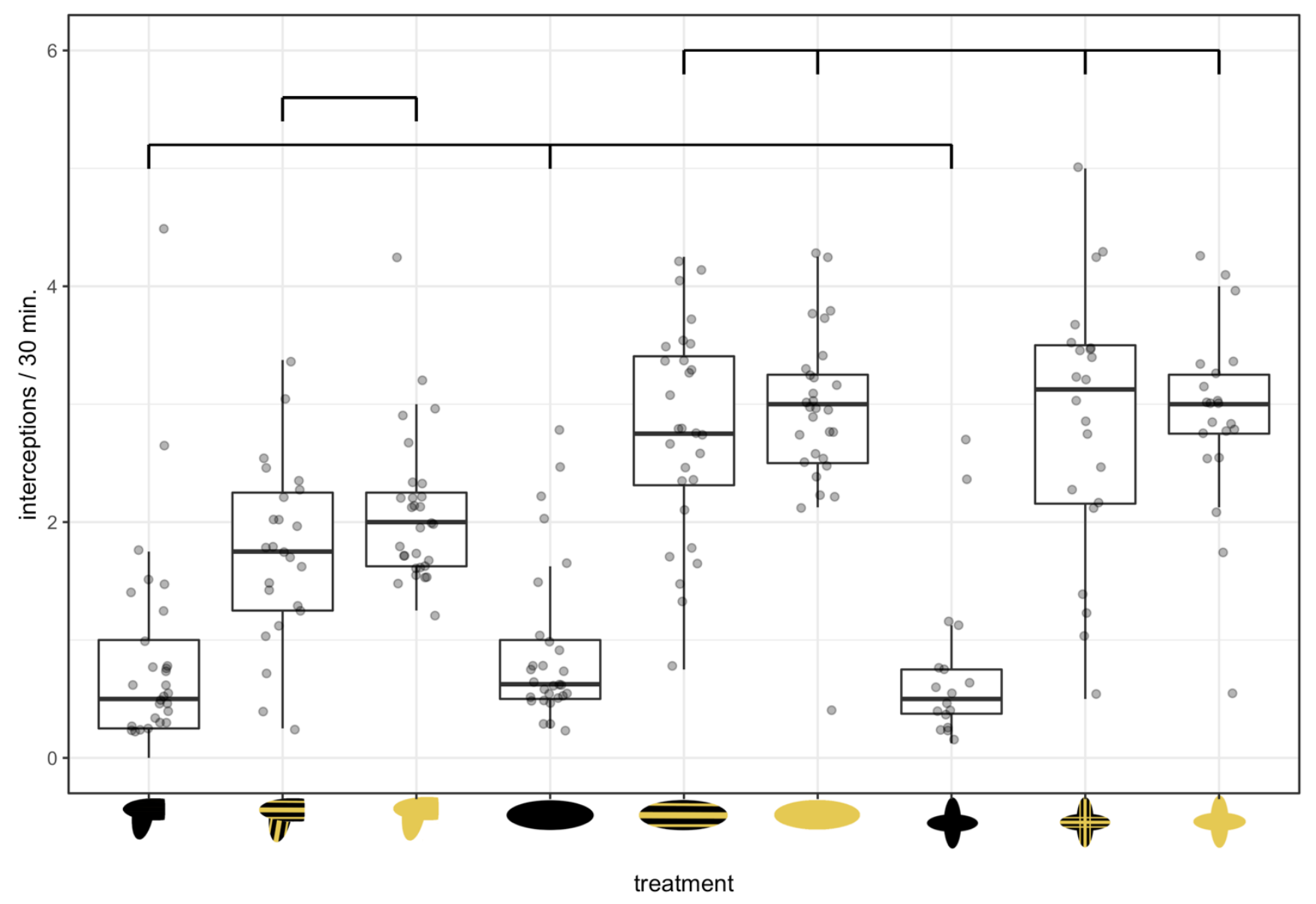

441 Figure 3: The effect of phenotypic manipulations (Fig. 1) on prey interception rates in $G$. 442 fornicata. Boxes denote the median and first and third quartiles, while whiskers extend to values 443 at a maximum of 1.5 times the inter-quartile range. Horizontal lines indicate statistically distinct 444 treatment groupings based on post-hoc multiple comparisons. Sample sizes, left-to-right; 29, 24, $44529,29,28,29,17,22,21$. 